\title{
MODELLING AND CONTROL OF OXYGEN PARTIAL PRESSURE IN AN UNDERWATER BREATHING APPARATUS WITH GAS RECYCLE
}

\author{
Franco Garofalo, Sabato Manfredi, Stefania Santini \\ Dipartimento di Informatica e Sistemistica, Università di Napoli Federico II \\ Via Claudio, 21 - 80125 Napoli, Italy
}

\begin{abstract}
The paper deals with the control of oxygen partial pressure in rebreather systems.

A new mathematical model describing the gas dynamics in the breathing system is developed and successively validated on experimental data. The model is consistent with the steady-state models commonly adopted for dive planning with rebreathers.
\end{abstract}

We show with some example how our model can be used to design control action and to evaluate control system performance.

KeyWords: control applications, underwater systems, modelling.

\section{Introduction}

Underwater breathing apparatus with the characteristics of partially or totally recycling the breathed gasses are commonly called 'rebreathers'. Differently from the well known open-circuit scuba, the exhaled gasses in rebreathers are not discarded in the form of bubbles but are recirculated into the re-breathing system, so achieving a tremendous increase of the efficiency of the underwater breathing apparatus.

Human respiration is a very complex phenomenon which involves many biochemical transformations. However the macroscopic chemical effects on breathed gasses are a (partial) oxygen subtraction and an increase of carbon dioxide with respect to the inhaled mixture. All other gasses different from oxygen are inert with respect to the respiration process and, hence, flow through the lungs without being chemically transformed. They are the greatest volumetric fraction of the breathing mixture. This means that a great part of exhaled gasses can be recycled (or better re-breathed), provided that oxygen be added to it and carbon dioxide be removed from it.

To recirculate the gasses, all rebreather design include a mouthpiece, through which the diver breathes, connected

${ }^{*}$ Corresponding author. Tel.: +39 081 7683845, Fax: +39081 7683816, e-mail: smanfredeunina.it with a collapsable bag that inflates when he exhales, and deflates when he inhales. This bag is usually called counterlung. In order to prevent the diver from inhaling the just exhaled gas, the rebreather is designed to force the gas circulation in one direction by using two one-way valves located on either side of the mouthpiece. The system formed by mouthpiece, connecting hoses, valves and the counterlung is often referred to as the "breathing loop'.

As already said, re-breathing in the loop produces an $\mathrm{O}_{2}$ decrease and a corresponding $\mathrm{CO}_{2}$ increase. Hence rebreathers have a scrubber for $\mathrm{CO}_{2}$ removal and a supply valve for $\mathrm{O}_{2}$ injection into the breathing loop. A possible classification of rebreathers can be made on the way they provide oxygen to the breathing loop [1].

Over-expansion of the counterlung when external pressure decreases is avoided by an overpressure relief valve and complete collapse of the counterlung when external pressure increases is avoided by the action of a demand valve which is triggered when the counterlung is almost completely collapsed. This always guarantees the availability to the diver of a minimum breathable gas volume at any depth. The schematics of a rebreather is given in figure 1.

The choice of the chemical nature and of the mix between inhert gasses used in a rebreather system has to do with decompression needs and to the narcotic effect of nitrogen, the natural respiration inhert gas contained in the air. These problems will not be addressed in this paper. We will focus, instead, our attention on oxygen level into the breathing loop. This is a crucial problem since to avoid hypoxia and hyperoxia [2], the oxygen level in the breathed mixture must be within specific limits. In particular, hypoxic symptoms appear when oxygen partial pressure falls down below 0.16 [atm] and oxygen toxicity symptoms are possible above 1.6 [atm] [3], [4]. So, oxygen partial pressures in the range 0.7-1.2[atm] are sufficiently far from these limits and can be considered safe for commercial, military or technical dives. 


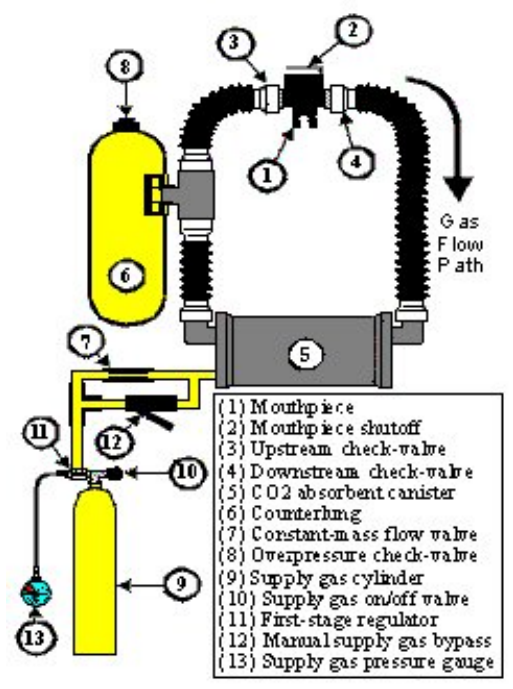

Fig. 1. Schematics of a rebreather.

Unfortunately $\mathrm{O}_{2}$ partial pressure in the breathing loop in subject to changes due to

- diver metabolism, which depends on the workload - internal pressure of the counter lung which changes with dive profile.

While variations of $\mathrm{O}_{2}$ partial pressure due to the depth can be easily predictable, the metabolic oxygen consumption rate can vary by a factor of 6 (or more) in normal conditions, and as much as 10 -fold in extreme conditions, depending on the activity level and cannot be easily predicted.

For these reasons controlling the oxygen partial pressure in a rebreather during all phases of a dive is a crucial task for the correct and safe use of this underwater breathing apparatus. It is common opinion that the diffusion of these devices, which are in principle much more efficient and safe with respect to the usual open circuit scuba system, will increase as soon as these control problems will be properly and reliably solved.

In next Section we develop a mathematical model of the dynamics of the gasses into the breathing loop. It is an important tool to understand the dynamic variations of oxygen partial pressures due to variations in depth and in workload during a dive. In successive sections, the mathematical model is used to construct a simulation model which can be used to analyse various dive scenarios and to test various control strategies that force the oxygen partial pressure to vary in a safe interval or to stay close a given set-point regardless of variation in metabolic oxygen needs and dive depth.

\section{Rebreather Mathematical Model}

An analytical model has been developed for predicting the oxygen level in rebreather for various mission profiles and diver activity levels.

The model considers the counterlung as adiabatic collapsable recipient which contain the breathable mixture with the following assumptions

- the breathable gas is made up of an inert gas (usually nitrogen and/or helium) and oxygen;

- the breathing gasses behave as perfect mixture;

- the recipient internal pressure instantaneously equals the external pressure;

- the respiration is simply modelled by a (partial) oxygen subtraction from the collapsable bag;

- the carbon dioxide scrubber behaves ideally, entrapping all produced $\mathrm{CO}_{2}$.

On the basis of these assumptions our model can derived by writing the perfect gas law for the two components of the breathing mixture

$$
\begin{aligned}
V_{\mathrm{O}_{2}} & =f\left(n_{\mathrm{O}_{2}}, p_{\mathrm{e}}\right)=\frac{n_{\mathrm{O}_{2}} R T}{p_{\mathrm{e}}}, \\
V_{\mathrm{I}} & =f\left(n_{\mathrm{I}}, p_{\mathrm{e}}\right)=\frac{n_{\mathrm{I}} R T}{p_{\mathrm{e}}},
\end{aligned}
$$

where $V_{\mathrm{O}_{2}}$ and $V_{\mathrm{I}}$ are the oxygen and inert partial volumes [liter]; $p_{\mathrm{e}}$ is the underwater external pressure [atm]; $T$ is the counterlung internal temperature $[\mathrm{K}] ; n_{\mathrm{O}_{2}}$ and $n_{\mathrm{I}}$ are the number of moles respectively of oxygen and inert in the counterlung. $R$ is the gas perfect law constant.

Obviously we can write

$$
\begin{aligned}
n_{\mathrm{O}_{2}} & =n_{\mathrm{O}_{2 \mathrm{~s}}}-n_{\mathrm{O}_{2 \mathrm{~m}}}, \\
n_{\mathrm{I}} & =n_{\mathrm{I}_{\mathrm{s}}},
\end{aligned}
$$

where subscript $S$ indicates the gas quantity injected by the supply valve and $n_{\mathrm{O}_{2 \mathrm{~m}}}$ is the metabolic oxygen consumption [mole].

Deriving equations (1) (2) with respect to time one obtains

$$
\begin{aligned}
\dot{V}_{\mathrm{O}_{2}} & =\frac{\dot{n}_{\mathrm{O}_{\mathrm{s}}} R T}{p_{\mathrm{e}}}-\frac{\dot{n}_{\mathrm{O}_{2 \mathrm{~m}}} R T}{p_{\mathrm{e}}}-\frac{n_{\mathrm{O}_{2}} R T}{p_{\mathrm{e}}{ }^{2}} \dot{p}_{\mathrm{e}} \\
\dot{V}_{\mathrm{I}} & =\frac{\dot{n}_{\mathrm{I}_{\mathrm{s}}} R T}{p_{\mathrm{e}}}-\frac{n_{\mathrm{I}} R T}{p_{\mathrm{ext}}{ }^{2}} \dot{p}_{\mathrm{e}}
\end{aligned}
$$

We stress that nominal metabolic oxygen consumption rate $\dot{n}_{\mathrm{O}_{2 \mathrm{~m}}}$ [mole/min] is variable with the workload but is independent from the external pressure. This is the 
main reason of the great efficiency of the rebreather system when compared with an open circuit scuba diving apparatus.

If the supply valve works at a constant mass flow, it is possible to write the dynamic model of counterlung in term of balance of volume flow rates [liter/min]

$$
\begin{aligned}
\dot{V}_{\mathrm{O}_{2}} & =\alpha \frac{p_{\mathrm{a}}}{p_{\mathrm{e}}} \mathrm{s}-\frac{p_{\mathrm{a}}}{p_{\mathrm{e}}} \mathrm{m}-V_{\mathrm{O}_{2}} \frac{\dot{p}_{\mathrm{e}}}{p_{\mathrm{e}}}, \\
\dot{V}_{\mathrm{I}} & =(1-\alpha) \frac{p_{\mathrm{a}}}{p_{\mathrm{e}}} \mathrm{s}-V_{\mathrm{I}} \frac{\dot{p}_{\mathrm{e}}}{p_{\mathrm{e}}},
\end{aligned}
$$

where $\mathrm{s}$ is the flow of the supply valve [liter/min], $\alpha$ is the oxygen fraction of the supplied gas mixture, $\mathrm{m}$ is the oxygen metabolic volume rate consumption [liter/min], and $p_{\mathrm{a}}$ is the pressure at the sea level [atm].

In this form, however, the counterlung model does not yet consider the actions of previously described demand and relief valves. Their actions can be modelled by introducing two more terms in balance equations (4) representing the demand valve flow $s_{d v}$ and the exhaust flow through the relief valve as $\mathrm{q}$. The oxygen fraction of the gas mixture supplied by the demand valve is $\beta$ while the gases in the mixture ( $\mathrm{I}$ and $\mathrm{O}_{2}$ ) are assumed to be vented out proportionally to their molar fraction.

With these two more terms the balance equations (4) become

$$
\begin{aligned}
& \dot{V}_{\mathrm{O}_{2}}=\alpha \frac{p_{\mathrm{a}}}{p_{\mathrm{e}}} \mathrm{s}-\frac{p_{\mathrm{a}}}{p_{\mathrm{e}}} \mathrm{m}-V_{\mathrm{O}_{2}} \frac{\dot{p}_{\mathrm{e}}}{p_{\mathrm{e}}}+\beta \mathrm{s}_{\mathrm{dv}}-\frac{V_{\mathrm{O}_{2}}}{V_{\mathrm{O}_{2}}+V_{\mathrm{I}}} \mathrm{q}, \\
& \dot{V}_{\mathrm{I}}=(1-\alpha) \frac{p_{\mathrm{a}}}{p_{\mathrm{e}}} \mathrm{s}-V_{\mathrm{I}} \frac{\dot{p}_{\mathrm{e}}}{p_{\mathrm{e}}}+(1-\beta) \mathrm{s}_{\mathrm{dv}}-\frac{V_{\mathrm{I}}}{V_{\mathrm{O}_{2}}+V_{\mathrm{I}}} \mathrm{q} .
\end{aligned}
$$

Since we are interested in the oxygen partial pressure $\mathrm{p}_{\mathrm{PO}_{2}}$ into the breathing loop, we can compute it from the partial volume values as

$$
\mathrm{p}_{\mathrm{p}_{2}}=\frac{V_{\mathrm{O}_{2}}}{V_{\mathrm{O}_{2}}+V_{\mathrm{I}}} p_{\mathrm{e}}
$$

As already seen the fundamental difference between various kinds of rebreather is mainly in how they supply gas to the breathing loop. Looking to equations ((5)-(6)) it is apparent that the model is easily adaptable to different rebreather system configurations just characterizing the injection system and the chemical content of the injected mixture.

\section{A. Model Validation on Experimental Data}

The model (equations (5)-(6)) has been validated through extensive simulations on experimental data col- lected during different dives. Data refer to a commercial semi-closed rebreather (SCR). The nominal rate of oxygen consumption is assumed to be 1 [liter/min].

As an example, in figures 2 the predicted oxygen partial pressure is compared to the measured pressure along the mission profile in figure 3 . The physical model shows a good agreement with the real scenario. Little discrepancies between the two plots obviously depend from the assumption of constant nominal metabolic rate and measurement uncertainties.

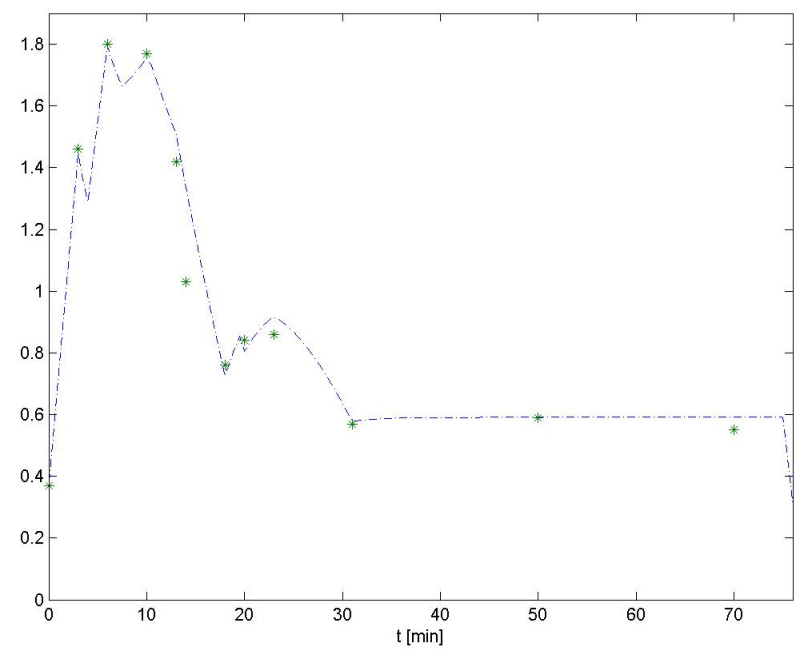

Fig. 2. Oxygen partial pressure in counterlung [atm]. Dotted line: predicted pressure. Stars: measured pressure.

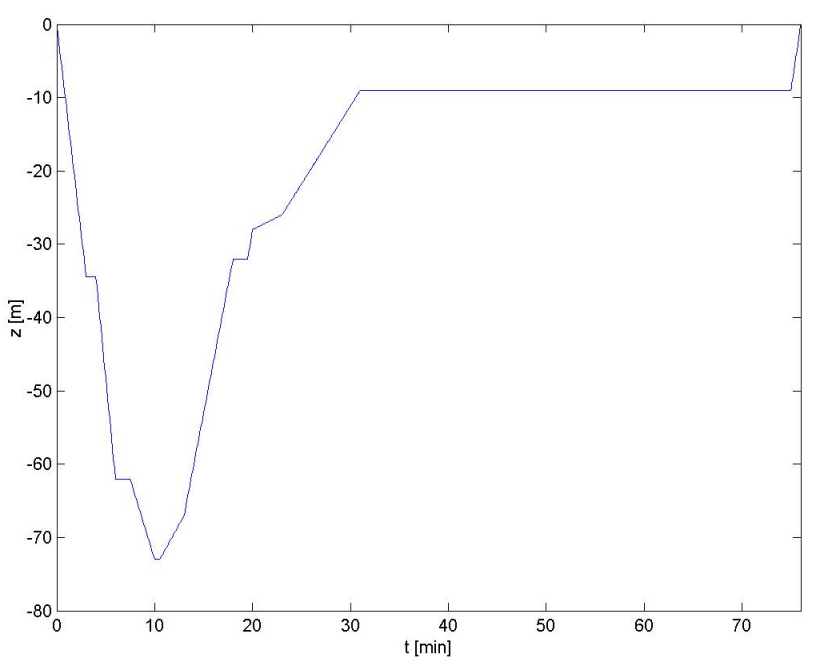

Fig. 3. Mission depth profile [meters].

Notice that during this mission, the diver has made a manual gas switch making a change in the supply gas from a rich (38\% oxygen fraction) to a lean (23\% oxygen fraction) mixture at the 3-th dive minute and conversely 
around the 18-th minute of the dive.

\section{Model-Based Control Strategies}

As already said, to minimize the risk of hypoxia and hyperoxia, a rebreather should guarantee relatively constant oxygen partial pressure in breathing loop in spite of variations in oxygen consumption and in depth.

We must consider however that the oxygen partial variability due to external pressure during ascent/descent can be easily computed (and hence compensated) from the knowledge of the depth and of the oxygen fraction in the supply gasses ( $\alpha$ and $\beta$ in equations (5)). The variations in metabolic oxygen needs, instead, vary from person to person especially during high exertion and cannot be easily predicted.

This means that we can plan a dive by selecting the right oxygen fraction in the supply mixture referring to nominal values of oxygen consumptions with the risk of exposing the diver to excessively low or high oxygen partial pressures.

Consider, for instance the mission profile given in figure 4 with a planned oxygen consumption of 1 [litre/min] and a real time-profile of oxygen consumption as that given in figure 5. This situation is rather usual, especially in a commercial dive, where the diver has a workload during the deeper phase of the dive.

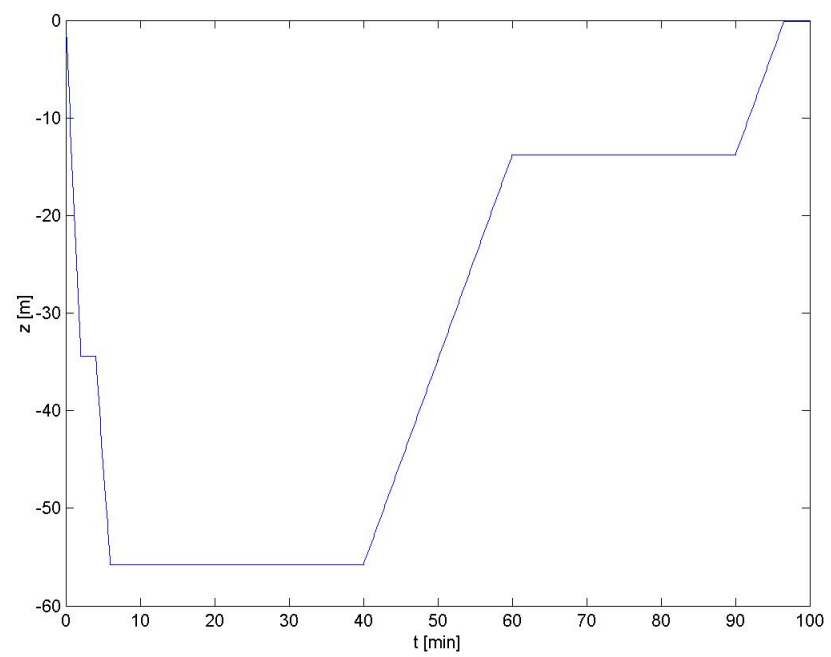

Fig. 4. Mission depth profile [meter].

Facing this dive with a $28 \%$ oxygen fraction breathing mixture and with a constant mass flow (typical injection of an SCR) fixed to 10 [sliter/min], the dynamics of the oxygen partial pressure in the loop, simulated with the aid of our model, is shown in figure 6 .

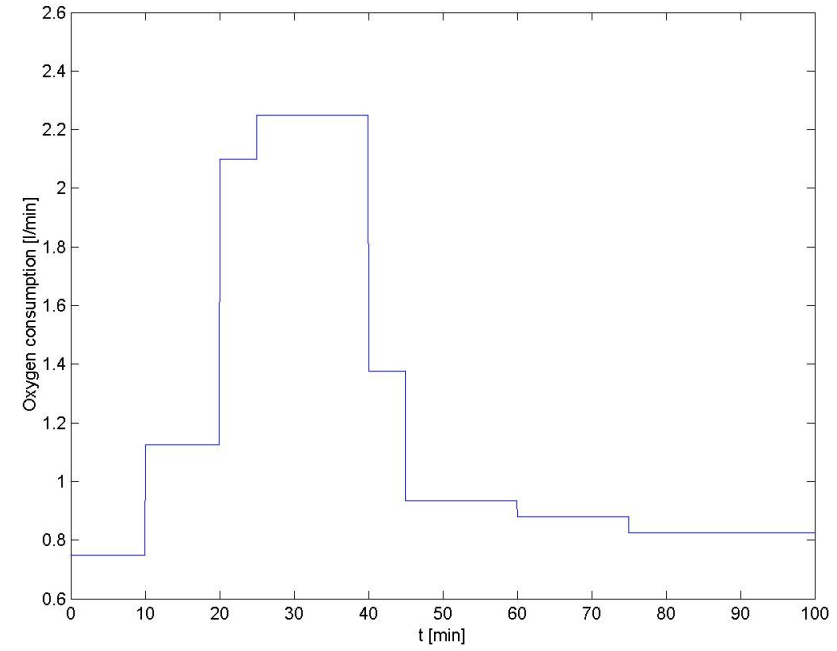

Fig. 5. Oxygen consumption during the mission [liter/min].

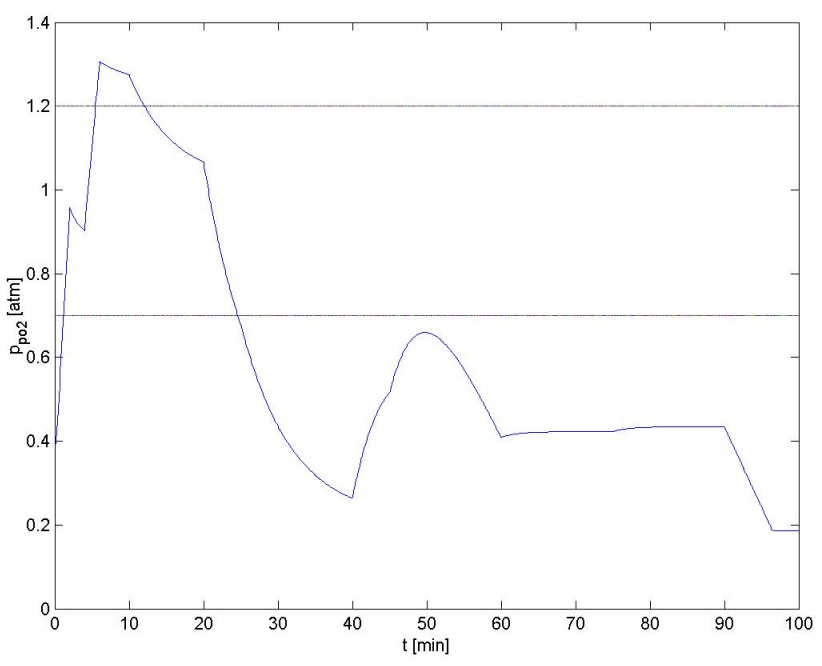

Fig. 6. Oxygen partial pressure dynamics corresponding to the depth profile in figure 4 . Lean mixture.

Instead, with a richer oxygen mixture (38\% oxygen fraction), we have the results shown in figure 7 .

In both cases the diver, especially during the deeper phase of the dive, is dangerously exposed to oxygen pressures out of the safety bounds.

To overcome this situation the diver could do a supply gas switch from rich to lean oxygen mixtures and viceversa. Our model can simulate this situation with a sudden change in the $\alpha$ parameter in equations (5) and the results are those given in figure 8 . Consider however that the switch time instant is very critical and can dramati- 


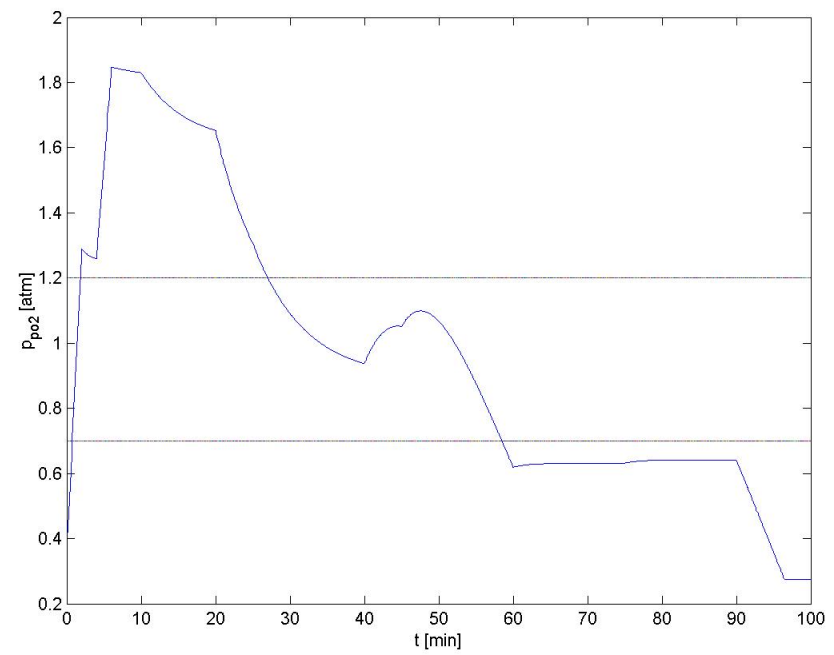

Fig. 7. Oxygen partial pressure dynamics corresponding to the depth profile in figure 4 . Reach mixture.

cally modify the $\mathrm{p}_{\mathrm{PO}_{2}}$ profile.

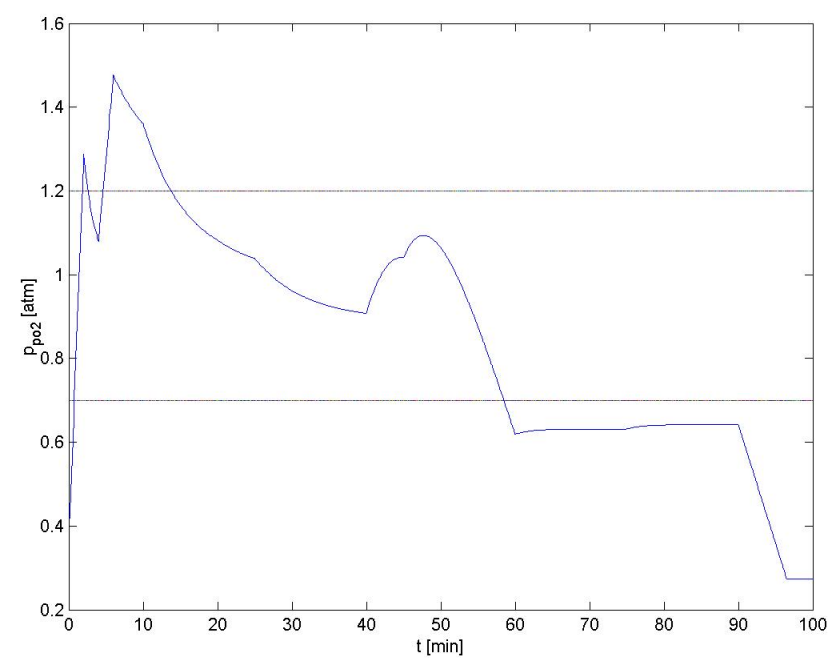

Fig. 8. Oxygen partial pressure dynamics corresponding to the depth profile in figure 4 with supply gas switches at the 3-th minute and at the 20-th minute of dive.

From the control point of view, in this case the diver operates a manual open-loop control of the oxygen partial pressure. Obviously better performances can be obtained by modulating the gas supply (the term $\mathrm{s}$ in model (5)) with respect to a measure of the number of breath per minute, which is an indirect estimate of the actual oxygen consumption.

The metabolic oxygen consumption can be considered as having a nominal constant component $\overline{\mathrm{m}}$ and a variable component $\Delta \mathrm{m}(t)$. In the same way control input can be considered as having a nominal component $\overline{\mathrm{s}}$ and $\mathrm{a}$ variable component $\Delta \mathrm{s}$. The nominal control component is designed to counteract the effect of the nominal disturbance in steady-state conditions. It can be evaluated as the solution of the following system, representing the steady-state behavior of equation (5)

$$
\begin{aligned}
0 & =\alpha \frac{p_{\mathrm{a}}}{p_{\mathrm{e}}} \overline{\mathrm{s}}-\frac{p_{\mathrm{a}}}{p_{\mathrm{e}}} \overline{\mathrm{m}}-V_{\mathrm{O}_{2}} \frac{\dot{p}_{\mathrm{e}}}{p_{\mathrm{e}}}-\gamma \overline{\mathrm{q}}, \\
0 & =(1-\alpha) \frac{p_{\mathrm{a}}}{p_{\mathrm{e}}} \overline{\mathrm{s}}-V_{\mathrm{I}} \frac{\dot{p}_{\mathrm{e}}}{p_{\mathrm{e}}}-(1-\gamma) \overline{\mathrm{q}},
\end{aligned}
$$

where $\bar{q}$ is the steady state exhaust from the counterlung, and $\gamma=\frac{V_{\mathrm{O}_{2}}}{V_{\mathrm{O}_{2}}+V_{\mathrm{I}}}$ is the steady-state oxygen fraction into the breathing loop.

By using the previous equation the nominal component of the control input turns out to be

$$
\overline{\mathrm{s}}=\frac{1-\gamma}{\alpha-\gamma} \overline{\mathrm{m}}
$$

from which the very well know formula that is commonly used for dive planning with SCR

$$
\gamma=\frac{\alpha \overline{\mathrm{s}}-\overline{\mathrm{m}}}{\overline{\mathrm{s}}-\overline{\mathrm{m}}} .
$$

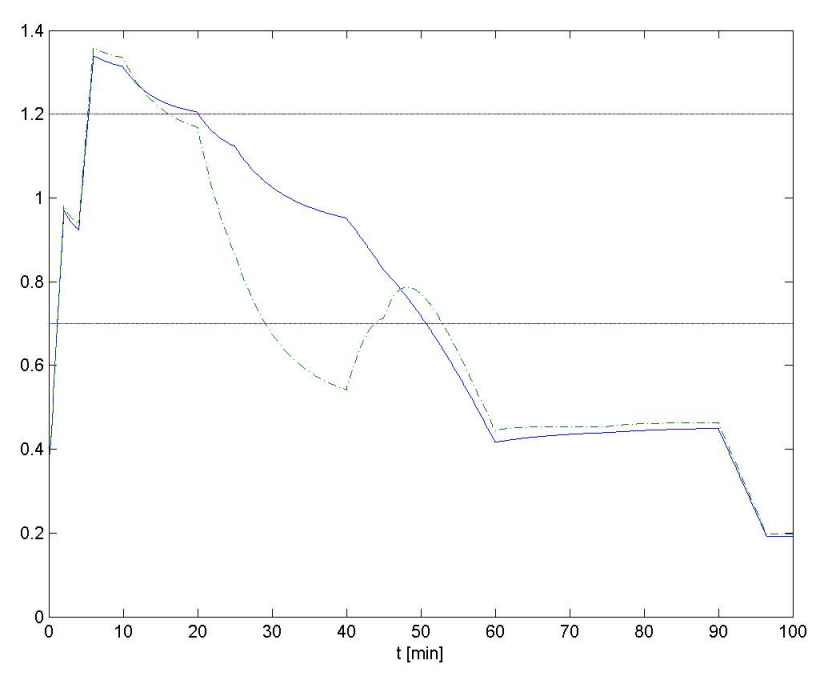

Fig. 9. Oxygen partial pressure dynamics corresponding to the depth profile in figure 4; controlled injection (solid line) and nominal injection (dotted line)

The metabolic oxygen consumption can be estimated on the base of the respiration frequency, bpm [number of breath/min], and of the tidal volume, tv, through the formula

$$
\mathrm{m}(t)=\epsilon(\operatorname{bpm}(t)) \times(\operatorname{tv}(t)),
$$




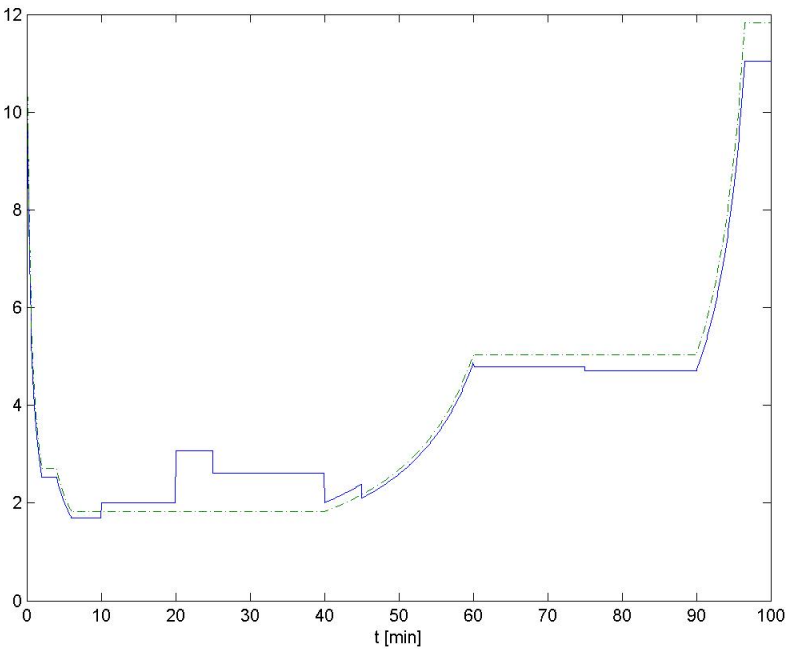

Fig. 10. Injected flow [liter/min] into the breathing loop; controlled injection (solid line) and nominal injection (dotted line).

where tv is the amount of gas moved in and out of the lungs with each breath [liter/breath], and $\epsilon$ represents the oxygen fraction burned in in one liter breath.

While the breathing frequency can be easily measured by a very simple and reliable counter located into the mouthpiece, the tidal volume can not be measured online. However tidal volumes lie into well known bounds, thus we can compute the feed-forward control action $\Delta \mathrm{m}(t)$ as

$$
\Delta \mathrm{m}(t)=g \epsilon(\operatorname{bpm}(t)-\overline{\mathrm{bpm}}) \times(\overline{\mathrm{tv}}),
$$

where $\overline{\mathrm{tv}}$ is the mean tidal volume value, $\overline{\mathrm{bpm}}$ is mean breathing frequency value, and $g$ is a constant gain.

Results of the control actions relative to the mission profile in figure 4 can be evaluated in figures 9-10. Simulations refer to a supply mixture having $\alpha=\beta=0.25$.

As expected oxygen partial pressure lies always the safe interval also during the heavy and deep part of the dive.

Future work will be addressed to the further validation of the proposed model on different rebreather systems, on the model-based design of simple and reliable feedback control strategies, and on field testing of all research work.

\section{Acknowledgments}

The authors would like to thank TECHNOSUB Ischia for the technical support provided during the experimen- tal part of this work.

\section{References}

[1] W. Skiles, "The rebreather revolution.," Rodale's Scuba Diving , pp. 91-100, 1991.

[2] J.M. Clark and C.J. Lambertsen, "Pulmonary oxygen toxicity: a review.," Pharmacol, pp. 37-133, 1971.

[3] C.J Lambertsen, "Effects of hyperoxia on organs and their tissues.," Extrapulmonary Manifestations of Respiratory Disease. Lung Biology in Health and Disease, Vol. 8. (E.D. Robin, ed.). Marcel Dekker, New York. pp. 239-303, 1978.

[4] J.M. Clark R. Gelfand Lambertsen, C.J., "Definition of tolerance to continuous hyperoxia in man. an abstract report of predictive studies.," Underwater and Hyperbaric Physiology IX (A.A. Bove, A.J. Bacherach, and L.J. Greenbaum, eds), Undersea and Hyperbaric Medical Society, Bethesda. pp. 717-735., 1987. 